DIACRONIE

\title{
Diacronie
}

Studi di Storia Contemporanea

$N^{\circ} 34,2$ | 2018

Scuola e società in Italia e Spagna tra Ottocento e Novecento

\section{Contro la guerra, contro il fascismo}

Clara Zetkin tra lotte e memoria

Jacopo Romano

\section{(2) OpenEdition}

\section{Journals}

\section{Edizione digitale}

URL: http://journals.openedition.org/diacronie/8574

DOI: 10.4000/diacronie.8574

ISSN: 2038-0925

Editore

Association culturelle Diacronie

\section{Notizia bibliografica digitale}

Jacopo Romano, «Contro la guerra, contro il fascismo », Diacronie [Online], N34, 2 | 2018, documento 11, Messo online il 29 juin 2018, consultato il 01 mai 2019. URL : http://journals.openedition.org/ diacronie/8574; DOI : 10.4000/diacronie.8574 


\section{Diacronie}

Studi di Storia Contemporanea

34, 2/2018

Scuola, politica e società in Europa tra Ottocento e Novecento

\section{Contro la guerra, contro il fascismo. Clara Zetkin tra lotte e memoria}

Jacopo ROMANO

Per citare questo articolo:

ROMANO, Jacopo, «Contro la guerra, contro il fascismo. Clara Zetkin tra lotte e memoria», Diacronie. Studi di Storia Contemporanea : Scuola, politica e società in Europa tra Ottocento e Novecento, 34, 2/2018, 29/06/2018,

URL: < http://www.studistorici.com/2018/06/29/romano_numero_34/ >

Diacronie Studi di Storia Contemporanea $\rightarrow$ http://www.diacronie.it

Rivista storica online. Uscita trimestrale.

redazione.diacronie@hotmail.it

Comitato di direzione: Naor Ben-Yehoyada - João Fábio Bertonha - Christopher Denis-Delacour - Maximiliano Fuentes Codera Anders Granås Kjøstvedt - John Paul Newman - Deborah Paci - Niccolò Pianciola - Spyridon Ploumidis - Wilko Graf Von Hardenberg

Comitato di redazione: Jacopo Bassi - Luca Bufarale - Gianluca Canè - Fausto Pietrancosta - Alessandro Salvador - Matteo Tomasoni - Luca Zuccolo

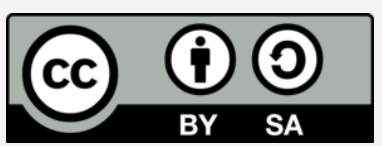

Diritti: gli articoli di Diacronie. Studi di Storia Contemporanea sono pubblicati sotto licenza Creative Commons 3.0. Possono essere riprodotti e modificati a patto di indicare eventuali modifiche dei contenuti, di riconoscere la paternità dell'opera e di condividerla allo stesso modo. La citazione di estratti è comunque sempre autorizzata, nei limiti previsti dalla legge. 


\section{1/ Contro la guerra, contro il fascismo. Clara Zetkin tra lotte e memoria}

Jacopo ROMANO

Questo lavoro si propone di analizzare alcuni aspetti dell'esistenza di Clara Zetkin (1857-1933). La militante tedesca femminista e socialista, allo scoppio della prima guerra mondiale, si distinse da gran parte del suo partito, la Spd, per la condanna della guerra e dei valori nazionalisti che portavano i popoli europei a combattersi tra loro nell'immane conflitto. All'indomani del conflitto, fino alla sua morte, Clara Zetkin lottò principalmente contro il fascismo e il nazionalsocialismo: in contrapposizione al nazismo, lei proponeva il sistema sovietico, che avrebbe liberato le masse dallo spettro di simili violenze e oppressioni. L'ultima parte del lavoro presenterà invece alcuni aspetti riguardanti l'immagine da lei lasciata in Occidente.

\section{Introduzione}

Clara Zetkin, nata Eisner (Wiederau, 5 luglio 1857 - Archangelskoje, 20 giugno 1933), è una figura poliedrica, della quale non è semplice fare un quadro completo ed una introduzione.

Consultando la sua pagina sulla Enciclopedia delle donne, si può leggere un punto che può aiutare in una estrema sintesi di quelli che furono gli obiettivi caratterizzanti la vita e le lotte di Clara: «La lotta per l'emancipazione di genere fu un punto di forza del suo pensiero come parte integrante del grande riscatto del proletariato cui si dedicò con grande passione» ${ }^{1}$.

La Zetkin si battè contro il liberalismo, l'imperialismo, il fascismo ed il nazismo. Si oppose alla prima guerra mondiale ed al sostegno del suo stesso partito, la Spd (Partito socialdemocratico tedesco), allo sforzo bellico ${ }^{2}$.

Questo lavoro si focalizzerà su alcuni aspetti in particolare della vita e della militanza di Clara Zetkin.

${ }^{1}$ SICARI RUFFO, Gaetanina, «Clara Zetkin», in Enciclopedia delle donne, URL:

$<$ http://www.enciclopediadelledonne.it/biografie/clara-zetkin/ > [consultato il 19 aprile 2018].

2 Ibidem. 
Il primo paragrafo cercherà di riassumere la sua vita ed il suo operato, citando anche alcune personalità di primo piano che ebbero modo di conoscerla, contrastandola o affiancandola. Tra questi vi furono Friedrich Engels, Vladimir Lenin, Rosa Luxemburg ${ }^{3}$.

Il rapporto con quest'ultima è particolarmente importante per l'oggetto del secondo paragrafo, l'antimilitarismo. Clara vedeva i proletari di tutti i paesi coinvolti nel primo conflitto mondiale come oppressi da un nazionalismo che li metteva in lotta tra loro, obbligandoli a combattere insieme ai loro «sfruttatori» ${ }^{4}$. Data la linea opposta del loro partito, Clara Zetkin e Rosa Luxemburg condivisero l'esperienza del carcere in questi anni ${ }^{5}$.

Il terzo paragrafo analizzerà le analisi e l'opposizione di Clara Zetkin al fascismo italiano e al nazionalsocialismo tedesco. Vi sono alcuni punti di continuità con il paragrafo precedente, dal momento che l'attivista evidenziò i nessi tra la prima guerra mondiale, la comparsa e lo sviluppo di queste formazioni sulla scena europea, ma non mancò di intercettare, e denunciare anche $\mathrm{i}$ caratteri nuovi e distintivi del caso hitleriano ${ }^{6}$ e di quello mussoliniano ${ }^{7}$

Il quarto ed ultimo paragrafo verte invece sulla memoria e la storiografia di Clara Zetkin in Occidente. Marilyn Boxer, introducendo Clara Zetkin. National and international contexts, sottolinea che «although once probably the best know woman socialist in the world, she remains so little know today, especially outside the former Soviet Union» ${ }^{8}$. Le immagini da lei lasciate ad alcune femministe, spesso di segno diverso, e le (scarse) tracce della sua memoria in movimenti comunisti e socialisti italiani oggi, caratterizzano la conclusione di questo lavoro.

Riguardo al materiale adoperato per questo lavoro, per il quale esprimo gratitudine alla Professoressa Eloisa Betti, è risultato molto importante per tutti gli aspetti il testo di Gilbert Badia, Clara Zetkin. Femminista senza frontiere, principale biografia sulla rivoluzionaria tedesca. Il testo di Boxer e Partington, più recente, è stato pure validissimo, in particolare per l'ultimo paragrafo.

Passando agli scritti di Clara Zetkin, i testi La questione femminile e la lotta al riformismo e Le sovversive, insieme al sito Marxists Internet Archive, permettono di leggere direttamente i suoi pensieri ed offrono quindi la possibilità di conoscere un punto di vista internazionalista, desideroso di pace, solidarietà, e lotta di classe in un mondo dominato dai nazionalismi e

\footnotetext{
${ }^{3}$ La Zetkin ebbe, sia con Lenin che con Rosa Luxemburg, rapporti molto importanti anche all'infuori della militanza politica, caratterizzati da stima e affetto. Cfr. ZETKIN, Clara, Note su Lenin, A.P.B., 1945, pp. 7-10 e 24.

4 GUTIÉRREZ, José, ALVAREZ, José, KLEISER B., Paul, Le sovversive, Roma, Erre emme, 1995, p. 189.

5 SICARI RUFFO, Gaetanina, «Clara Zetkin», in Enciclopedia delle donne, URL:

< http://www.enciclopediadelledonne.it/biografie/clara-zetkin/> [consultato il 19 aprile 2018].

${ }^{6}$ Cfr. BADIA, Gilbert, Clara Zetkin. Femminista senza frontiere, Roma, Erre emme, 1994, pp. 275-286.

7 RAGIONIERI, Ernesto, Italia giudicata 1861-1945, ovvero la storia degli italiani vista da altri, Bari, Laterza, 1969, p. 534.

8 BOXER, Marilyn J., PARTINGTON, John S., (a cura di), Clara Zetkin. National and international contexts, London, Socialist History Society, 2013, p. 3.
} 
sconvolto dalla prima guerra mondiale. Il testo di Ernesto Ragionieri contiene invece le analisi della Zetkin sul fascismo italiano.

\section{Cenni Biografici}

Clara Eisner nacque nella cittadina sassone di Widerau, nel 1857. Durante le scuole magistrali, che frequentò dai 17 ai 21anni, a Lipsia, entrò in contatto con alcuni studenti russi, tra cui Ossip Zetkin (suo futuro marito), che militava nella socialdemocrazia tedesca. In seguito alle repressioni antisocialiste, avviate dal cancelliere Otto von Bismarck a partire dal 1878, sia lei che Zetkin lasciarono la Germania, ritrovandosi a Parigi nel 1882, dove si sposarono nel novembre dello stesso anno ${ }^{9}$. Nella capitale francese la Zetkin conobbe importanti marxisti come Jules Guesde, profughi socialisti e anarchici di vari paesi europei e prese parte a diverse manifestazioni operaie $^{10}$. Nonostante gli ostacoli legislativi, il socialismo cresceva in Germania e in altri paesi europei.

Nel 1889 venne fondata a Parigi la II Internazionale, che per Zetkin rappresentò «il primo intervento importante sulla scena internazionale del movimento operaio e l'avvio di [un'] organizzazione internazionale del movimento femminile proletario» ${ }^{11}$. Clara diede un apporto centrale allo sviluppo dell'Internazionale fino allo scoppio della prima guerra mondiale, contribuendo sia ai lavori preparatori sia come delegata delle donne socialiste berlinesi ${ }^{12}$. Nel 1890, dopo la revoca delle leggi antisocialiste, fece ritorno in Germania, per diventare, un anno dopo, redattrice del giornale socialdemocratico «Die Gleicheit» [L'uguaglianza].

Nel 1893, in occasione del III Congresso della II Internazionale, incontrò Engels, che divenne un suo estimatore ${ }^{13}$. Nel 1896 diede un contributo importante al Congresso di Gotha della Spd. Nel corso di altri convegni di partito (nel 1898 e nel 1899), lei e Rosa Luxembourg lottarono molto energicamente per condurre il loro partito politico a contrastare ed ostacolare la corrente riformista di Eduard Bernstein ${ }^{14}$. Per la Zetkin, quest'ultimo:

rigetta il concetto di catastrofe dell'ordinamento sociale ed economico del capitalismo quale presupposto imprescindibile per [la] società socialista e spera in un graduale, frammentario contrabbando del socialismo dentro la società capitalistica attraverso riforme sociali, nascita

\footnotetext{
9 ZETKIN, Clara, La questione femminile e la lotta al riformismo, Milano, Mazzotta, 1977, pp. 3-4.

10 Ibidem.

${ }^{11}$ Ibidem, pp. 4-5.

12 Ibidem.

13 Ibidem.

14 Ibidem, pp. 5-6.
} 
di sindacati [...] non tanto un mezzo per abbattere il capitalismo, quanto invece un mezzo per rafforzarlo attraverso l'imborghesimento del proletariato ${ }^{15}$.

Con l'avanzare dello spettro della prima guerra mondiale, Clara Zetkin abbracciò l'antimilitarismo, opponendosi anche a esponenti del suo stesso partito che si dichiaravano disponibili a votare i crediti di guerra e a «battersi in difesa della patria» ${ }^{16}$. Durante il primo conflitto mondiale si avvicinò quindi ai dissidenti spartachisti e, dal 1919, incontrò più volte Lenin (che conosceva da oltre un decennio) ${ }^{17}$. Il pensiero del rivoluzionario russo era visto dalla Zetkin come particolarmente vicino non solo per gli ideali comunisti, ma anche per quanto concerneva i diritti delle donne. Il leader comunista, oltre a ritenere l'eguaglianza della donna come carattere imprescindibile del comunismo, era per Clara anche un uomo consapevole dell'importanza che ricoprivano le donne nelle attività comuniste e di quanto la loro inclusione fosse per certi aspetti determinante. Nel gennaio 1925, ad un anno dalla morte di Lenin, Clara Zetkin cominciava uno scritto di alcune pagine in questo modo:

\begin{abstract}
Il compagno Lenin mi ha spesso parlato della questione femminile. Le riconosceva una grande importanza, poiché il movimento femminile era per lui parte costitutiva e, in certe condizioni, parte decisiva del movimento delle masse. È inutile dire che egli considerava la piena eguaglianza sociale della donna come un principio indiscutibile del comunismo. [...] Ero entusiasta di tutto quello che le donne russe avevano fatto durante la rivoluzione, di tutto quello che ancora facevano per difenderla e per aiutarla a svilupparsi. Quanto alla posizione e all'attività delle donne nel partito bolscevico, mi sembrava che, da questo lato, il partito si mostrasse all'altezza del suo compito ${ }^{18}$.
\end{abstract}

Con la comparsa del fascismo, divenne presidente del Soccorso Rosso Internazionale, nel 1924, strumento mondiale deputato ad assistere le «vittime della reazione e del fascismo»" ${ }^{19}$. Il 30 agosto del 1932, in quanto deputata più anziana, tenne il discorso di apertura del Reichstag, prima che Hermann Göring ne assumesse la presidenza. Lanciò un appello a comunisti e socialdemocratici: unirsi in un unico fronte per contrastare il partito nazionalsocialista ${ }^{20}$. Questo discorso dimostrò il coraggio e le convinzioni di Clara, che non si tirò indietro di fronte al pericolo nazista, nonostante la forza di quest'ultimo e la sua pericolosità. Infatti, alle elezioni del 31 luglio 1932, il Partito nazionalsocialista passò da 107 seggi di 2 anni prima a 230. La Zetkin, pur non partecipando alla campagna elettorale, venne eletta nella lista della Kpd (Partito comunista di Germania) del

\footnotetext{
15 Ibidem, p. 142.

16 BADIA, Gilbert, op. cit., p. 139.

17 ZETKIN, Clara, La questione femminile e la lotta al riformismo, cit., pp. 7-8.

18 ZETKIN, Clara, Lenin e il movimento femminile, Roma, Centro Rosso, 1977.

19 ZETKIN, Clara, La questione femminile e la lotta al riformismo, cit., p. 8.

20 GUTIÉRREZ, José, ALVAREZ, José, KLEISER B., Paul, op. cit., p. 195.
} 
Württemberg. Quell'anno fu caratterizzato, ogni domenica, da scontri tra le SA naziste e i militanti di sinistra. Alla Kpd arrivò una lettera, firmata «Un nazionalsocialista», che minacciava direttamente la Zetkin: se lei avesse avuto «l'impudenza di inaugurare la seduta del Reichstag» sarebbe stata afferrata "per le orecchie» e cacciata dal Reichstag «a pedate» ${ }^{21}$. I giornali nazionalsocialisti e di destra scrivevano di lei accusandola di alto tradimento, nominandola con espressioni come «ebrea comunista» e «moscovita». Il giornale di Goebbels utilizzava il termine «onnaccia» ${ }^{22}$.

Lei tornò da Mosca alla fine di agosto, molto debole e quasi cieca. Il 30, in un parlamento pieno di deputati nazionalsocialisti con le uniformi delle SA e delle SS, fece un discorso in cui denunciava il «terrore» nazista, la «codardia» dei liberali e «la passività di gran parte dei lavoratori». La soluzione per una Germania in preda al nazismo era, per Clara, il modello sovietico, dove i lavoratori erano abbastanza maturi da aver creato «un nuovo ordine economico al riparo dalle crisi disastrose», grazie alla eliminazione della proprietà dei mezzi di produzione, che era la «causa del sistema anarchico di produzione» ${ }^{23}$. Nonostante questa difesa del comunismo sovietico, quello che per Badia fu il punto «più alto» del discorso al Reichstag consistette nell'appello a mettere «in secondo piano le differenze politiche, sindacali, religiose ed ideologiche» per contrastare il «fascismo» e le «organizzazioni degli schiavi dello sfruttamento». Rivolgendosi alle donne, ancora gravate dalle «catene della schiavitù del loro sesso», disse che dovevano prestare il loro contributo a questo fronte unificato. Anche un giornale di destra riconobbe coerenza in questo suo discorso, discorso che venne ascoltato da «Amici e nemici»: era necessario «sopportarla anche se ha convinzioni inaccettabili» ${ }^{24}$. Decise poi di ritornare in Unione Sovietica, dove morì il 20 giugno del 1933 in un sanatorio ad Archangelskoje, vicino a Mosca ${ }^{25}$. I suoi resti vennero tumulati nel Cremlino ${ }^{26}$.

\section{L'antimilitarismo}

Dal 1890 al primo dopoguerra, l'esperienza politica di Clara Zetkin fu contraddistinta dall'antimilitarismo, nonostante la politica della socialdemocrazia tedesca andasse in un'altra direzione. Mentre i socialdemocratici mostrarono una "complicità» verso l'imperialismo tedesco ${ }^{27}$, Clara Zetkin manifestava le sue opinioni contro la Seconda guerra boera (1899-1902) in

\footnotetext{
${ }^{21}$ BADIA, Gilbert, op. cit., p. 283.

22 Ibidem.

23 Ibidem, p. 284.

24 Ibidem, pp. 284-285.

25 ZETKIN, Clara, La questione femminile e la lotta al riformismo, cit., p. 8.

${ }^{26}$ GUTIÉRREZ, José, ALVAREZ, José, KLEISER B., Paul, op. cit., p. 196.

27ZETKIN, Clara, La questione femminile e la lotta al riformismo, cit., p. 7.
} 
Gran Bretagna, conflitto che fu sostenuto anche da alcuni socialisti. Frase chiave del suo intervento fu «Guerra contro la guerra», vale a dire una chiamata all'azione piuttosto che ad una opposizione passiva ${ }^{28}$.

La sua visione era originale anche rispetto ai socialisti che, come lei, si opponevano alle guerre, perché andava oltre gli schemi ideologici e integrava la questione con argomentazioni di genere. La civiltà non rappresentava le realizzazioni di una elite istruita, ma era insita nella funzione biologica delle donne. La perdita dei mariti, figli e fratelli rendeva le donne vittime della guerra: «Women who have worked and wept refuse to give up their loved ones to be shamelessly and callously slaughtered through the frenzy of the military and Imperialist factions ${ }^{29}$.

Nel 1907 ebbe uno scontro molto acceso con Gustav Noske quando egli, nel corso del Congresso di Essen, esternò la sua disponibilità a combattere per il proprio paese minacciato ${ }^{30}$. A Copenhagen, nel 1910, fece passare una risoluzione che sottolineava il dovere, da parte di «tutte le compagne», di ricordare le risoluzioni ostili al conflitto votate al Congresso internazionale di Stoccarda e di educare i figli alla pace ${ }^{31}$. Effettivamente, nel corso del Congresso del 1907, Rosa Luxemburg, Vladimir Lenin e Julij Osipovic Cederbaum Martov avevano fatto passare un emendamento, a cui si oppose la maggioranza dei delegati tedeschi, che prevedeva, nel caso di conflitto, una opposizione dei socialdemocratici allo stesso e di «sfruttare la violenta crisi economica e politica prodotta dalla guerra per sollevare la popolazione» e velocizzare «l'abolizione del dominio di classe capitalistico»"

Per il $1^{\circ}$ maggio del 1913, Clara Zetkin scrisse un editoriale dal titolo Noi siamo la forza che vedeva come obiettivo principe della manifestazione la denuncia della corsa agli armamenti e della propaganda favorevole alla guerra ${ }^{33}$. Nel dicembre dello stesso anno, i suoi energici pensieri internazionalisti di pace e fratellanza comparvero sulle pagine di «The Labour Woman ${ }^{34} »$. Si tratta di un documento che, almeno per chi scrive, rende davvero in modo eccezionale le consapevolezze della Zetkin sugli effetti catastrofici di un conflitto internazionale, sui progressi letali di alcune armi che contraddistingueranno la Prima guerra mondiale e sull'odio che il nazionalismo riusciva a veicolare in numerosi paesi. Il suo intervento, vero e proprio appello alle socialiste britanniche, aveva l'emblematico titolo «German Women to Their Sisters in Great Britain». Aveva agito come segretaria internazionale delle donne socialiste, ed a nome delle

\footnotetext{
28 BOXER, Marilyn S, PARTINGTON, John S (a cura di), op. cit., p. 23.

29 Ibidem, pp. 24-25.

30 BADIA, Gilbert, op. cit., p. 139.

31 Ibidem

32 Ibidem, pp. 137-138.

33 Ibidem, p. 139.

34 ZETKIN, Clara, O'Callaghan, Einde (traduzione di), «German Women to Their Sisters in Great Britain», in Marxists Internet Archive, <URL: https://www.marxists.org/archive/zetkin/1913/12/sisters.htm > [consultato il 15 settembre 2017].
} 
socialiste tedesche inviava alle socialiste britanniche un messaggio di pace, fraternità e libertà. Il testo proseguiva citando le guerre nei Balcani, che erano ancora fresche negli animi di lei e delle sue compagne: il sangue di uomini che si assassinavano tra loro con armi da fuoco, città e villaggi devastati dalle fiamme, mutilazioni, i pianti di madri, sorelle e bambini, privati dei loro legami affettivi e di un sostegno per la famiglia albergavano dentro di loro ${ }^{35}$.

Gli uomini erano qui definiti «bread-winners», per sottolineare anche i problemi economici che sorgevano nelle famiglie che li perdevano al fronte. Proseguiva sottolineando che le popolazioni europee si trovavano sempre più vicine al «terrible abyss of a gigantic war, such as the world never has seen before $»^{36}$. Le classi dirigenti del continente sprecavano infatti il denaro sottratto alle tasche dei lavoratori nelle caserme, artiglieria da campo e navale, e per ogni altro più possibile perfetto strumento volto all'assassinio e alla distruzione massificate in terra, cielo e aria $^{37}$. Migliaia di giovani venivano esercitati per essere $\mathrm{i}$ "Cains of their brethren abroads» ${ }^{38}$. Come fanno, si chiedeva, le classi dirigenti a convincere le masse che sacrifici umani e materiali siano necessari? Propagandando continuamente la necessità dell'odio tra le nazioni e quanto sia interesse vitale per le stesse disporre di eserciti e marine da guerra numerosi. Proseguiva affermando che i tedeschi, come i britannici, non dovevano essere identificati con gli «UpperTen», dagli ufficiali agli industriali bellici che hanno interesse nella guerra, ma con i milioni di uomini e donne che facevano una vita dura e che stavano giungendo alla consapevolezza che i nemici non erano fuori dai confini, ma radicati nelle istituzioni capitalistiche che li governavano ${ }^{39}$.

In conclusione, la militante tedesca esortava a combattere contro tutti i pregiudizi nazionalisti e ad una «holy war» volta ad emancipare i lavoratori. Se il capitalismo era una guerra sociale di tutti contro tutti, la guerra di classe dei lavoratori significava la fraternità tra i lavoratori di tutti i paesi $^{40}$.

I mesi che precedettero l'agosto del 1914 videro, in opposizione al diffuso nazionalismo favorevole alla guerra, molte attività di segno contrario da parte della Zetkin e della fazione socialdemocratica più di sinistra ${ }^{41}$. Come emerge dal testo Le sovversive, infatti, i socialdemocratici in Germania (come negli altri paesi) abbracciarono in maggioranza il "socialpatriottismo" nel 1914. Il suo obiettivo divenne quello di contrastare questa svolta socialdemocratica e di battersi

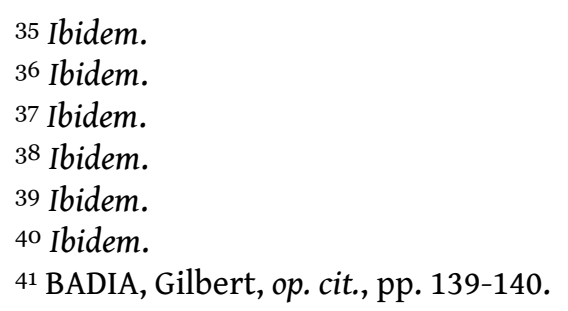


per la salvaguardia dei valori rivoluzionari ed internazionalisti ${ }^{42}$. Raggiunse così Bruxelles alla fine di luglio, per prendere parte alla riunione dell'Ufficio politico del socialismo internazionale e tentare di ottenere dai delegati iniziative importanti, ma non ebbe seguito. Tornata in Germania seppe dell'ordine del Kaiser di mobilitazione generale e, il 4 agosto, fu informata telefonicamente che la Spd aveva votato i crediti di guerra. Mesi dopo scrisse che la notizia la portò al pensiero del suicidio ${ }^{43}$.

La votazione della Spd provava per lei che «l'imperialismo ha piegato al servizio dei propri obiettivi tutte le forze del proletariato». Scrisse della censura e della svolta «nazionalista e sciovinista» di gran parte di stampa socialdemocratica, pur sentendosi sorvegliata dalle autorità. Resisteva con il suo giornale "Gleicheit», controllato e ostacolato nelle uscite e distribuzioni ${ }^{44}$.

Anche alcuni anni dopo, in occasione del XVIII Congresso del Partito Socialista Italiano, la Zetkin non mancò di condannare l'operato di numerosi socialisti nel 1914. Quell'anno si verificò quello che lei definì «il passaggio della socialdemocrazia dal campo del proletariato internazionale, della rivoluzione, nel campo degli imperialisti nazionali, della "difesa della patria!"» ${ }^{45}$. La socialdemocrazia divenne, per lei, un partito "borghese" votato alle riforme sociali. Le risoluzioni della II Internazionale, comprese quelle dei Congressi di Stoccarda, Copenhagen e Basilea volte ad ostacolare il conflitto mondiale divennero, allo scoppio dello stesso, dei «Pezzi di carta senza valore ${ }^{46}$.

Che impatto ebbe il conflitto sui legami di Clara Zetkin con le donne socialiste di altre nazionalità?

John Partignon ha lavorato sulle stampe socialiste e laburiste britanniche riguardanti due congressi socialisti per la pace $(1912,1915)$ che ebbero un apporto fondamentale di Clara Zetkin. Lei era in quel periodo segretaria della Socialist Women's International, antimilitarista e socialista nella Germania nemica dei britannici: rappresentava per molte socialiste britanniche contrarie alla guerra «the focal point», una voce di buonsenso in contesto di crisi internazionali e prova vivente "that comradship could traverse the battlefronts» ${ }^{47}$. A guerra appena scoppiata, pensò subito a convocare in clandestinità una conferenza internazionale delle donne e, supportata da compagne straniere, fece arrivare messaggi a socialiste di vari paesi. Si incontrarono con lei a Berna donne provenienti da Germania, Gran Bretagna, Russia, Italia, Polonia e Svizzera ${ }^{48}$. Lei e la

\footnotetext{
$4^{2}$ GUTIÉRREZ, José, ALVAREZ, José, KLEISER B., Paul, op. cit., p. 188.

43 BADIA, Gilbert, op. cit., pp. 139-141.

44 Ibidem, pp. 142-143.

45 ZETKIN, Clara (introduzione di), WALECKI, Enrico (conclusione di), Il Partito socialista italiano sulla via del riformismo. Discorsi dei delegati della Internazionale comunista al 18. Congresso del PSI, Roma, Libreria editrice del Partito comunista d'Italia, 1921, pp. 34-35. Le virgolette sono presenti nel testo. Cfr. Ibidem, p. 35.

46 Ibidem, p. 35.

47 BOXER, Marilyn J., PARTINGTON, Johns S. (a cura di), op. cit., p. 22.

$4^{8}$ BADIA, Gilbert, op. cit., pp. 144-145.
} 
maggioranza delle delegate (a differenza delle russe e delle polacche) adottarono una risoluzione che condannava il conflitto senza esplicitare una condanna per i partiti socialisti che aderivano ad esso. All'unanimità passò un appello alle donne di tutti i paesi in guerra, scritto dalla stessa Zetkin e poi fatto circolare clandestinamente in tutti i paesi coinvolti. Nel testo rappresentava gli uomini combattenti come zittiti, snaturati e privati della coscienza da parte del capitalismo dedito alla «conquista di territori», che mascherava la sua guerra con la difesa della patria. Era compito delle donne, per il bene dei loro cari costretti a morire e a essere uccisi, agire contro il «capitalismo e le sue stragi di esseri umani sacrificati alla ricchezza e al potere dei possidenti!» ${ }^{49}$. La conferenza fece incontrare per la prima volta le socialiste dei paesi in guerra e incoraggiò tutte le pacifiste ${ }^{50}$. Il «Labour Leader» fu il primo giornale a riportare la dichiarazione congiunta della Conferenza di Berna che, pur essendo frutto della sensibilità di differenti partecipanti, fece emergere le posizioni della Zetkin ${ }^{51}$. Tale dichiarazione sottolineava che la guerra aveva annullato inestimabili doni della civiltà e che aveva ostacolato il progresso dei più nobili ideali con delle barriere di barbarie $^{52}$. Alla fine del conflitto, i paesi si sarebbero trovati prosciugati delle loro forze vitali, con le loro risorse economiche decimate e i loro progressi in campo sociale ritardati in modo indeterminato. Riferendosi alla propaganda nazionalista, la dichiarazione aggiungeva che "Calumnies and insults dishonour each nation, and hide the gifts wich each has given to the common civilisation of the world $»^{53}$.

Per la prima volta dall'inizio della guerra, veniva fatto esplicito riferimento all'interruzione della lotta di classe, operata dal conflitto mondiale, che aveva sostituito la fratellanza internazionale dei lavoratori con un fratricidio internazionale ${ }^{54}$.

Come nel precedente riferimento alle guerre nei Balcani, l'antimilitarismo della Zetkin (e delle sue compagne straniere) non si limitava quindi ad analizzare e condannare le i tratti distintivi della guerra moderna come le morti, le mutilazioni, le devastazioni, ma anche quello che la stessa comportava in campo sociale, economico e nell'animo dell'umanità globalmente intesa.

La dichiarazione della Conferenza di Berna indicava alcune proposte per pacificare l'Europa. La ricetta consisteva in una pace senza annessioni o conquiste, in un riconoscimento dei diritti di popoli e dei paesi indipendentemente dalle loro dimensioni. La giustizia doveva occuparsi di punire determinate responsabilità dei belligeranti, senza però sfociare in umiliazioni: «[...] no

\footnotetext{
49 Ibidem, p. 146.

50 Ibidem, p. 147.

${ }^{51}$ BOXER, Marilyn J, PARTINGTON, Johns S (a cura di), op. cit., p. 30.

${ }^{2}$ Il testo, in lingue inglese, riporta le dichiarazioni della stessa Zetkin. Cfr. Ibidem, pp. 30-31.

53 Ibidem, pp. 30-31.

54 Ibidem, p. 31.
} 
humiliating and insupportable conditions upon any country and expiation of the wrong inflicted upon Belgium ${ }^{55}$.

Nel 1915, con il raffreddamento dell'entusiasmo nazionalista in Germania, molte donne, private anche del salario del marito al fronte, tornarono a lavorare nelle fabbriche. Furono le prime a manifestare, il 18 marzo del 1915, davanti al Reichstag ${ }^{56}$. Cominciò a farsi strada tra loro l'idea che la guerra e i maggiori oneri che ricadevano su di loro fossero in parte dovuti a errori o all'assenza dei "compagni maschi". I testi della Zetkin accentuarono il loro carattere femminista con lo scoppio del conflitto mondiale ${ }^{57}$ e le socialdemocratiche manifestarono contro la linea del partito, che rispose il 15 febbraio del 1915 con lo scioglimento dell'ufficio femminile.

Anche le autorità si preoccuparono della Zetkin e, il 20 luglio, l'attivista venne arrestata con l'accusa di "alto tradimento". Fu interrogata sull'appello di Berna. Il partito inviò uno dei suoi due presidenti, Hugo Haase, a difenderla. Clara venne così rilasciata il 12 ottobre dello stesso anno, ufficialmente per motivi di salute, anche se stava relativamente bene. Secondo alcuni vi fu un patto tra la direzione della Spd e i pubblici poteri, poiché i secondi erano spaventati dal seguito che Clara aveva sulle donne e dalla possibilità che le iniziative per la sua scarcerazione aumentassero $^{58}$. La Zetkin visse la detenzione nella prigione di Karlsruhe come periodo transitorio e naturale della propria battaglia, dopo il quale ricominciare a combattere ${ }^{59}$. Anche dopo la scarcerazione, come ha messo in luce Gilbert Badia analizzando la corrispondenza Clara Zetkin negli archivi berlinesi, lei veniva ogni tanto sorvegliata da spie, sia in orari diurni che notturni ${ }^{60}$.

Nel 1917 si trovò costretta a lasciare la direzione del giornale "Gleicheit», a causa della incompatibilità delle sue posizioni ed azioni con la linea di partito ${ }^{61}$. La salute cagionevole non le impedì di partecipare, nel novembre del 1918, ai movimenti che portarono all'abolizione della monarchia e alla proclamazione della repubblica ${ }^{62}$. Il 17 novembre, pochi giorni dopo aver partecipato ai moti, scrisse a Rosa Luxemburg il suo punto di vista su quegli eventi. Riconosceva che la rivoluzione era partita da soldati e aveva rivendicazioni militari, ma essa si era, a suo avviso, evoluta in una «lotta politica rivoluzionaria contro il militarismo, contro il regime personale, per la democrazia politica».

\footnotetext{
55 Ibidem Parlando del Belgio, fa con probabilità riferimento all'invasione del paese, da parte tedesca, nel 1914. L'invasione avvenne ai danni di un paese neutrale. Cfr. Ibidem.

${ }^{56}$ BADIA, Gilbert, op. cit., pp. 148-149.

57 Ibidem, pp. 149-150.

58 Ibidem, pp. 151-152.

59 GUTIÉRREZ, José, ALVAREZ, José, KLEISER B., Paul, op. cit., pp. 189-190.

60 BADIA, Gilbert, op. cit., pp. 153-154.

${ }^{61}$ GUTIÉRREZ, José, ALVAREZ, José, KLEISER B., Paul, op. cit., p. 190.

62 Ibidem, p. 191.
} 
La lettera proseguiva sottolineando come il militarismo e il «regime personale» non fossero più nemici ma "pilastri ben custoditi dell'ordine borghese»" ${ }^{63}$ Esattamente una settimana dopo, era invece Rosa Luxemburg a scrivere all'amica e compagna, confermandole la possibilità, precedentemente richiesta dalla Zetkin, di scrivere volantini per mobilitare le donne. Nella lettera la Luxemburg raccomandò a Clara Zetkin di essere breve con i testi, a causa della carenza di carta, e di parlare genericamente delle lavoratrici e della rivoluzione ${ }^{64}$. Un'altra lettera, datata 11 gennaio 1919, sempre scritta da Rosa Luxemburg alla Zetkin, ricordava la diversità degli spartachisti dal vecchio partito e la loro genuinità ${ }^{65}$. Solo pochi giorni dopo questa lettera, il 15 gennaio del 1919, Rosa Luxemburg e Karl Liebknecht furono catturati dai miliziani nazionalisti dei Freicorps, che poi li assassinarono con un colpo alla nuca. I circoli di destra avevano messo da tempo una taglia su di loro, ma anche la stampa socialdemocratica esprimeva disprezzo verso i due attivisti. Karl Kautsky e Bernstein dichiararono che i due erano responsabili della loro sorte atroce $^{66}$.

\section{Contro i fascismi}

Dagli anni del primo dopoguerra fino alla sua morte, avvenuta nel 1933, l'attivista tedesca dedicò le sue energie alla lotta contro i fascismi, il fascismo italiano prima e il nascente nazionalsocialismo tedesco poi. Nel mutato contesto politico, Clara sottolineò le analogie con la guerra del 1914-1918 e il militarismo che aveva lungamente combattuto.

Con la guerra, infatti, larghi strati della piccola e media borghesia si erano trovati «proletarizzati»; i piccoli contadini vertevano in condizioni disperate; ingegneri, liberi professionisti, scrittori e artisti erano in preda alla «miseria nera», mentre ufficiali e sottufficiali si trovarono disoccupati con la fine delle ostilità ${ }^{67}$. Tutti cercavano nuovi impieghi fissi e nuove posizioni sociali.

Ingrossavano le file dei fascisti «uomini e donne delusi dal mancato rinnovamento sociale conseguente ai grandi massacri del $1914-1918 »^{68}$. Clara Zetkin definiva il fascismo come la «decomposizione dello Stato borghese» per eccellenza, causata dalle crisi del capitalismo. Il fascismo non si reggeva su caste elitarie ma su larghi strati sociali. Non andava confuso, come

\footnotetext{
63 Ibidem, pp. 197-198 L'espressione «regime personale» potrebbe qui alludere alla monarchia tedesca impersonata dal Kaiser Guglielmo II. Si tratta infatti di uno scritto del 17 novembre 1918 sulla Rivoluzione tedesca che, in quei giorni, portò alla abdicazione del Kaiser. Cfr. Ibidem.

64 LUXEMBURG, Rosa, BASSO, Lelio (a cura di), Lettere 1893-1919, Roma, Editori Riuniti, 1979, p. 262.

65 Ibidem, p. 265.

${ }^{66}$ GUTIÉRREZ, José, ALVAREZ, José, KLEISER B., Paul, op. cit., p. 227.

67 BADIA, Gilbert, op. cit., p. 278.

68 Ibidem, pp. 278-279.
} 
facevano (a suo parere) i socialisti riformisti, per una semplice reazione al bolscevismo sovietico ${ }^{69}$. Il fallimento dei comunisti in Europa facilitò, secondo lei, le adesioni a questi nuovi movimenti. Molte persone, secondo la Zetkin, non videro più il proletariato agente trasformatore della società, ma trasferirono la loro fiducia negli «individui più capaci, più energici, più intrepidi di tutte le classi, che si debbono raccogliere insieme in una comunità. Per i fascisti tale comunità è la nazione.... ${ }^{70}$.

La Zetkin si pronunciò anche nello specifico sul caso italiano, evidenziando come la grande guerra avesse facilitato e fosse alla base delle esperienze dei fascisti. L'industria pesante del settentrione, sorta celermente, aveva assicurato profitti straordinari grazie alla prima guerra mondiale ma, a causa della mancanza di carbone e acciaio nel paese, la sua crescita svanì in fretta, causando una crisi in campo industriale, commerciale e artigianale. Grandi masse di lavoratori e lavoratrici persero il lavoro, che si aggiungevano ai reduci in cerca di lavoro, ai mutilati, agli orfani e alle vedove bisognosi di assistenza. Fu questo il fertile terreno per Benito Mussolini, che aveva attraversato il «fiume di sangue della guerra mondiale» secondo Clara Zetkin ${ }^{71}$. Per lui, passato dal socialismo pacifista all'interventismo, il conflitto avrebbe fatto dell'Italia un paese capitalistico moderno. Nel 1919, Mussolini fondò a Milano il primo fascio di combattimento per garantire «agli eroi delle trincee ed ai lavoratori i frutti rivoluzionari di una guerra rivoluzionaria $»^{72}$. Nel marzo del 1932, annotando in una lettera che il successo del nazionalsocialismo in Germania non era stato arginato dai comunisti, Clara rifletteva sul fatto che erano molti i proletari nazisti, delusi sia dai socialdemocratici che dai comunisti tedeschi. Tanti disoccupati militavano anche nei corpi paramilitari hitleriani ${ }^{73}$.

Lo Stato autoritario era la creatura e lo strumento dei fascisti volto a realizzare il loro ideale. Essi si dotarono di un programma pseudorivoluzionario per catturare l'attenzione delle masse ed i loro desideri e, allo stesso tempo, di gruppi paramilitari. Queste formazioni, sia nel contesto mussoliniano che in quello hitleriano, divennero ausiliari della polizia una volta che i loro capi presero il potere ${ }^{74}$. Clara Zetkin ammetteva la presenza di elementi rivoluzionari e antiborghesi all'interno del fascismo, ma li vedeva comunque come «prigionieri» di quelli reazionari. Nella sua analisi, la Zetkin sembra prevedere quello che accadde poco dopo la sua morte, il 30 giugno del 1934: l'eliminazione delle squadre d'assalto naziste, le SA, da parte hitleriana ${ }^{75}$.

\footnotetext{
69 Ibidem.

70 Ibidem, p. 279.

${ }^{71}$ RAGIONIERI, Ernesto, Italia giudicata 1861-1945, ovvero la storia degli italiani vista da altri, Bari, Laterza, 1969, pp. 536-537.

72 Ibidem, p. 537

73 BADIA, Gilbert, op. cit., p. 282.

74 Ibidem, p. 280

75 Ibidem, p. 279
} 
Tornando alle riflessioni sul fascismo in Italia, Clara Zetkin sottolineava come, esso, una volta giunto al governo, avesse tradito il suo programma originario. I fascisti avevano richiesto di rivedere i contratti statali per le forniture di guerra e l'avocazione allo Stato stesso dell' $85 \%$ dei profitti di guerra ma, una volta al governo, le loro politiche andarono in direzione opposta ${ }^{76}$. Il parlamento insediò una commissione deputata all'esame dei contratti per le forniture di guerra, che avrebbe dovuto riferire alla Camera. Mussolini, con una delle sue prime decisioni, obbligò la commissione a riferire direttamente a lui e introdusse la reclusione fino a 6 mesi per la divulgazione dei risultati dell'indagine ${ }^{77}$. Il provvedimento salvò dalla compromissione numerosi capitalisti dell'industria pesante, che di Mussolini erano protettori e finanziatori. Le loro attività ricevevano miliardi dal regime per le loro forniture, mentre sulla «confisca dei profitti di guerra tacciono tutti i flauti fascisti $»^{78}$. Da un punto di vista militare, i fascisti si erano precedentemente dichiarati desiderosi di innovazione: abolizione dell'esercito permanente, accorciamento del servizio, limitazione dell'esercito alla difesa del paese e non più a guerre imperialiste erano alcuni dei loro punti. In seguito, oltre alla mancata abolizione dell'esercito permanente vi fu un aumento del tempo di servizio dagli 8 ai 18 mesi $^{79}$. Aumentava, inoltre, il numero degli ufficiali di carriera. Gli armamenti, proseguiva la Zetkin, venivano potenziati in vista di grosse campagne imperialistiche: l'artiglieria, la flotta e l'aviazione conobbero uno sviluppo «eccezionale». Erano stati commissionati numerosi sommergibili ed incrociatori per la marina, mentre l'aviazione si apprestava ad incamerare nuovi aerei e nuove basi. L'industria pesante ricevette molto denaro per la produzione degli «apparecchi più moderni e di strumenti militari di sterminio» ${ }^{80}$.

Questo intervento della Zetkin è del 1924. Leggerlo non permette solo di toccare con mano la sua coerenza nella lotta contro tutto ciò che è bellicista, nazionalista, e pronto ad intercettare bisogni e desideri di ampi strati di popolazione per ottenerne il consenso, con programmi in gran parte modificati all'indomani della salita al potere. Esso mostra ancora una volta la sua lungimiranza. Alcuni anni dopo queste sue riflessioni, una volta che lei era deceduta, le nuove armi del regime furono protagoniste della campagna etiopica. Ancora dopo, la corsa agli armamenti ed il progresso tecnologico in campo bellico caratterizzarono il secondo conflitto mondiale. Armi molto potenti e letali uccisero, mutilarono e ferirono milioni di persone, sia militari che civili.

\footnotetext{
${ }^{76}$ RAGIONIERI, Ernesto, op. cit., p. 546.

77 Ibidem.

78 Ibidem.

79 Ibidem, p. 547.

80 Ibidem, pp. 547-548.
} 


\section{L'eredità di Clara Zetkin in Occidente tra storiografia e memoria}

Qual è stata l'eredità di Clara Zetkin in Occidente? Per rispondere alla domanda ci pare utile esaminare sia l'ambito storiografico che quello legato alla memoria.

Sul piano storiografico, la biografia redatta da Gilbert Badia all'inizio degli anni Novanta in francese, tradotta in più lingue, rimane la principale. Badia consultò gli archivi berlinesi ed ebbe accesso sia a documenti originali che a copie di lettere, di cui parte degli originali si trovavano invece a Mosca. Altre lettere furono da lui consultate sempre a Berlino, a Karlsruhe e ad Amsterdam. Il testo di Badia fornisce anche utili indicazioni sulla bibliografia circolante sulla Zetkin. Pur non esistendo una raccolta completa delle opere della Zetkin, ci sono raccolte e antologie di testi in lingua tedesca ${ }^{81}$, più volte ristampati. La rivista della ex Berlino Est, la «Beiträge zur Geschichte der Arbeiterbewegung» (BZG) [Contributi alla storia del movimento operaio], ha pubblicato molti suoi scritti. Tra questi ultimi, alcune lettere inaccessibili fino al 1989. Badia indicava poi alcune sue opere pubblicate in francese ed in italiano. Passando alle opere su di lei, Badia ne elencava 14, compreso il suo. Quest'ultimo e un altro sono stati pubblicati in francese, uno in inglese e tutti gli altri in tedesco ${ }^{82}$.

Lo stesso Badia, introducendo il suo lavoro, sottolineava che aveva l'obiettivo di mostrare alcuni lati della Zetkin spesso sconosciuti e travisati. Per la fase della vita della Zetkin che si conclude con il 1919, in entrambe le Germanie e nei paesi anglosassoni non sono mancati studi sulle sue idee e sul suo ruolo. Per la fase successiva, che vide Clara Zetkin avvicinarsi e soggiornare ripetutamente in Urss, le opere pubblicate nella Repubblica federale tedesca, negli Stati Uniti e in Gran Bretagna, furono estremamente limitate negli anni della Guerra Fredda. Nella

\footnotetext{
${ }^{81}$ BADIA, Gilbert, op. cit., pp. 306-307 Il testo che ha avuto il maggior numero di ristampe è Erinnnerungen an Lenin [Ricordi di Lenin]. Tra gli altri testi elencati da Badia, ci sono le antologie tematiche su Clara Zur Theorie und Tatkik der kommunistschen Bewegung [Sulla teorie e la tattica del movimento comunista] e Kunst und Proletariat [Arte e proletariato]. Viene ricordata anche la Ausgewählte Reden und Schriften [Discorsi e scritti scelti], considerata dall'autore la più ricca raccolta di testi della Zetkin. Cfr. Ibidem.

82 Ibidem, pp. 307-310 I testi in lingua francese che raccolgono articoli, opuscoli ed estratti di Clara Zetkin sono Batailles pour les femmes [Battaglia a favore delle donne] e Les batailles révolutionaires de l'Allemagne [Battaglie rivoluzionarie in Germania]. I testi in lingua italiana elencati da Badia sono stati in buona parte utilizzati per questo articolo: Il Partito socialista italiano sulla via del riformismo, Italia giudicata e La questione femminile e la lotta al riformismo. Passando all'elenco delle 14 opere su Clara Zetkin, alcune di queste sono: Die Frauenfrage in Lichte des Sozialismus [La questione femminile alla luce del socialismo] di Anna Blos; Anpassung als Emanzipation Zur Theorie und Praxis der bürgerlichen Fraunbewegung in Deutschland. 1894-1933 [Adattamento come emancipazione. Sulla teoria e la prassi del movimento borghese delle donne in Germania. 1894-1933] di Barbara Greven-Aschoff; Contribution à l'histoire des femmes allemandes entre 1848 et 1920 à travers les itinéraires de Louise Otto, Helene Lange, Clara Zetkin et Lily Braun [Contributo alla storia delle donne tedeschetra il 1848 e il 1920-attraverso gli itinerari di L. Otto, H. Lange, C.Zetkin e L. Braun] di Marianne Walle. Cfr. Ibidem.
} 
Repubblica democratica tedesca vennero invece si pubblicate ed in numero significativo, ma diversi aspetti non vennero toccati ${ }^{83}$.

Il discorso sulla storiografia si intreccia con quello della memoria. Passando al più recente libro di Partington e Boxer, del 2013, vi è un capitolo dal titolo molto significativo «Defamed in the West, Idealised in the East? On the Reception of Clara Zetkin in Germany after $1945 »^{84}$. Il capitolo sostiene la tesi che Clara Zetkin è recepita come una rappresentante del movimento delle donne proletarie, come socialista, comunista e antimilitarista, ma in pochi hanno realizzato che fu una delle maggiori iniziatrici della Giornata Internazionale della donna, oggi celebrata da sindacati e movimenti femministi ${ }^{85}$. Nella Repubblica Federale Tedesca, durante la Guerra Fredda, dagli anni cinquanta all'inizio dei sessanta, era vista come "persona non grata» ${ }^{86}$ e la Giornata Internazionale della donna veniva considerata come «event of the devil» ${ }^{87}$. La Giornata veniva commemorata nel paese solo da poche formazioni, che ricordavano anche la Zetkin stessa. Tra questi vi erano il «Demokratischer Frauenbund Deutschlands» [Lega delle donne democratiche di Germania], messo fuori legge nel 1957, e la «Westdeutsche Frauenfriensbewegung» [Movimento della Germania Ovest delle donne per la pace] ${ }^{88}$.

Alla fine degli anni sessanta, la sua figura venne riscoperta da diverse anime del femminismo, che offrirono letture diverse. Parte di loro la ritenevano come responsabile della scissione del movimento femminista tra fine Ottocento e inizio Novecento. La giornalista Schwarzer, nel 1975, descrisse la separazione del movimento in borghesi e proletarie come illegittima, perché negava le specifiche discriminazioni di cui era vittima il genere femminile e riduceva la questione femminile alle lotte di classe. Un'altra giornalista, Marielouise Janssen-Jurreit, scrisse nel suo best-seller Sexismus. Über die Abtreibung der Frauenfrage che ella «refused the struggle of the sexes and fought after 1889 against feminism and feminists ${ }^{89}$. A questo riguardo, sono interessanti anche le descrizioni dell'operato della Zetkin in due diversi testi, uno del 1996 e l'altro del 2011. Karen Hunt scrisse che il contributo della Zetkin sulla questione femminile era volto a evidenziare unicamente le relazioni tra genere e classe sociale. Clara Zetkin, nel suo opuscolo The Question of Women Workers and Women at the Present del 1889, indicò che gli interessi delle donne proletarie coincidevano con la loro classe sociale e non con i movimenti femministi di estrazione borghese. In quello stesso anno, durante il suo intervento all'Internazionale di Parigi, la Zetkin ribadì che le

\footnotetext{
83 Ibidem, pp. 11-12.

84 Cfr BOXER, Marilyn J., PARTINGTON, John S. (a cura di), op. cit., pp. 45-53, p. 45.

85 BOXER, Marilyn J., PARTINGTON, John S. (a cura di), op. cit., p .45.

86 Nel testo. Cfr BOXER, Marilyn J., PARTINGTON, John S. (a cura di), op. cit., p .45.

87 Ibidem.

88 Ibidem.

89 Ibidem, p. 46 Marielouise Jeannsen-Jurreit, Sexismus. Über die Abtreibung der Frauenfrage (Sexism: On the miscarriage of the woman question), Munich, 1976 Cfr. Ibidem, p. 108.
} 
donne lavoratrici non dovevano aspettarsi «nothing» dai movimenti femministi borghesi ${ }^{90}$. In The contested nation. Ethnicity, class, religion and gender in national histories, menzionando proprio Clara Zetkin e Rosa Luxemburg, si dice che per le militanti comuniste i diritti delle donne erano un obiettivo per cui dover lottare, ma tale lotta era vista come parte della più ampia lotta di classe ${ }^{91}$. Ma non mancarono letture diverse.

Alcuni movimenti femministi-socialisti, come «Aktionsrat zur Befreiung der Frau» [Consiglio d'azione per la liberazione delle donne], a Berlino Ovest, e «Arbeitskreis Emanzipation» [Gruppo di lavoro per l'emancipazione], a Bonn, alla fine degli anni sessanta, abbracciarono la Giornata della donna e cominciarono a studiare e discutere scritti marxisti, compresi quelli della Zetkin. Apprezzarono e riconobbero come attuali le sue posizioni sul lavoro femminile, considerando che all'epoca il pensiero dominante relegava le donne al ruolo di casalinghe ${ }^{92}$. Nel 1981, la famosa scrittrice Gisela Elsner mise in discussione la tesi secondo la quale Clara Zetkin era responsabile della scissione del movimento femminista, sottolineando che aveva riconosciuto alcune idee progressiste delle femministe borghesi in materia di voto e di lavoro femminile ${ }^{93}$. Il partito socialdemocratico tedesco la ignorò, mentre quello comunista organizzò due conferenze, rispettivamente nel 1980 e nel 1983, per il cinquantenario della morte di Clara Zetkin e per il suo $130^{\circ}$ anniversario di nascita ${ }^{94}$.

Quando nel 1993, pochi anni dopo la riunificazione della Germania, in Francia uscì il libro di Badia, molte case editrici tedesche si rifiutarono di tradurlo, ritenendo il soggetto incapace di suscitare interesse. Venne comunque tradotto l'anno seguente dalla berlinese Dietz. Le recensioni tedesche, all'opposto di quelle francesi, furono molto negative ${ }^{95}$.

Per quanto riguarda la memoria di Clara Zetkin in Italia, negli ultimi anni, nel nostro territorio, due partiti hanno deciso di intitolare proprio a lei due loro sezioni. Nel 2014, ad Erice, è stata dedicata la sezione delle donne del PSI. L'articolo la cita unicamente come «esponente politica tedesca che nei primi del '900 si batté per i diritti delle donne $»^{96}$. Nel 2015 una scelta analoga è stata presa dal Partito Comunista dei Lavoratori di Brescia ${ }^{97}$.

\footnotetext{
90 HUNT, Karen, Equivocal feminists. The Social Democratic Federation and the woman question 1884-1911, Cambridge: Cambridge University, 1996, pp. 63-64 Il titolo dell'opuscolo della Zetkin è indicato in inglese nel testo della Hunt. Cfr. Ibidem, p. 63.

${ }^{11}$ BERGER, Stefan, LORENZ-BAGINSTOKE, Chris, The contested nation. Ethnicity, class, religion and gender in national histories, New York, Palgrave Macmillan, 2011, p. 222.

92 BOXER, Marilyn S, PARTINGTON, John S (a cura di), op. cit., p. 46.

93 Ibidem, p. 47.

94 Ibidem.

95 Ibidem, pp. 50-51.

96 «Erice, "Clara Zetkin" è la prima sede in Italia delle donne socialiste», in Avantil, 27 giugno 2014, URL:

< http://www.avantionline.it/2014/06/erice-clara-zetkin-la-prima-sede-in-italia-delle-donne-socialiste/\#.WdFg_1u0PIV > [consultato il 29 settembre 2017].

97 «Presentazione della sezione "Clara Zetkin" del Partito Comunista dei Lavoratori di Brescia», in Partito Comunista dei Lavoratori, URL: < http://www.pclavoratori.it/files/index.php?obj=NEWS\&oid=4397 > [consultato il 29 settembre 2017].
} 


\section{L'AUTORE}

Jacopo ROMANO ha conseguito la laurea triennale in Storia presso l'Università di Bologna con una tesi dal titolo Tecnologia bellica e reparti indigeni nelle colonie italiane: da Adwa alla Guerra d'Etiopia, relatrice Prof.ssa Karin Pallaver. Attualmente è iscritto al corso di laurea magistrale in Scienze Storiche dell'Università di Bologna.

URL: < http://www.studistorici.com/progett/autori/\#Romano > 